\title{
COMPARISON BETWEEN MEASUREMENTS AND WRF NUMERICAL SIMULATION OF GLOBAL SOLAR IRRADIATION IN ROMANIA
}

\author{
Dragos Isvoranu ${ }^{1}$, Viorel Badescu ${ }^{2}$ \\ ${ }^{1}$ Dept. of Aerospace Sciences "Elie Carafoli", Univ. Politehnica of Bucharest, Str. Gh. Polizu 1, 011061, \\ Bucharest, ROMANIA \\ ${ }^{2}$ Candida Oancea Institute, Univ. Politehnica of Bucharest, Spl. Independentei 313, 060042 Bucharest, \\ ROMANIA
}

\begin{abstract}
Article Info
Received: 17.03.2013

Accepted: 29.03.2013

Keywords: global irradiance forecast, NWP simulation, PV stations

PACS: $96.60 . \mathrm{Ub}, 88.40 \mathrm{mr}$, 02.60.-x, 92.60.Wc
\end{abstract}

\begin{abstract}
The paper presents a comparative analysis between the surface global irradiation measured for Romania and the predicted irradiation obtained by numerical simulation. The measured data came from the Romanian National meteorological Administration. Based on a preliminary analysis that took into account several criteria among which, performance, cost, popularity and meteorological and satellite data accessibility we concluded that a combination GFS-WRF(NMM) or GFS-WRF(ARW) is most suitable for short term global solar irradiation forecasting in order to assess the performance of the photovoltaic power stations (Badescu and Dumitrescu, 2012, [1], Martin et al., 2011, [2]).
\end{abstract}

\section{Introduction}

Solar energy has a specific common characteristic: a high variability in space and time. It is highly dependent on the cloud structure, day/night cycles, the humidity and the aerosol load of the atmosphere. Due to intermittent weather patterns, solar energy power plants cannot guarantee the amount of energy which is requested by the electrical grid operators in order to respond at any time to the end users demands. Therefore, secondary energy sources have to be considered locally or on a regional scale. Additionally, weather has a major impact on the electricity transmission and distribution grids, from the risk of outages and transmission capacity on one side and to the end users highly variable and weather dependant demands on the other side.

An increased production of solar energy will lead to a higher number of dispersed production sites with a highly variable weather dependant energy production which is fed into the medium and lower voltage grids. In such systems, production forecasts for the next minutes up to several days ahead are of high importance as they enable the utilities and the 
grid operators to adapt the load schedule, hence to contribute to an optimized production and distribution of solar energy and make the PV stations more competitive against classical production sources. In this sense, accurate forecasts of solar energy production as well as an optimized implementation of these forecasts for load schedules are of utmost importance. The objective of the present paper is the assessment of the quality and performance of global solar irradiance forecasting. The paper presents a comparative analysis between the surface global irradiance measured for Romania and the predicted irradiance obtained by numerical simulation. The measured data comes from the Romanian National Meteorological Administration.

\section{Solar radiation forecasting}

The basic idea of numerical weather prediction is to sample the state of the atmosphere at a given time and use the equations of fluid dynamics and thermodynamics to estimate the state of the fluid at some time in the future. Models are initialized using this observed data. The irregularly spaced observations are processed by data assimilation and objective analysis methods, which perform quality control and obtain values at locations usable by the model's mathematical algorithms (usually an evenly spaced grid).

Our primarily research interest lies within the first three meteorological forecasting ranges: now-casting, that is a 0-3 hours description of forecasted weather parameters; very short-range weather prediction (up to 12 hours prediction); Short-range weather forecasting (beyond 12 hours and up to 72 hours description of weather parameters); Medium-range weather forecasting (beyond 72 hours and up to 240 hours description of weather parameters).

Now-casting systems use the combination of radar extrapolation techniques with satellite and Numerical Weather Prediction (NWP) model products to produce an extended short-period forecast. The coordination of power generation by weather-dependent renewable energies (e.g., wind and solar) and reserve power sources (e.g., gas turbines or hydro) can be closely controlled in real-time based on weather observations and short-term forecasts.

In the very short range forecasting domain, numerical weather models are coupled with post-processing modules in combination with real-time measurements data assimilation. In the short and medium range forecasting time domains only the numerical weather model in combination with post-processing modules and satellite information is applied.

Solar yield forecast is still on an early state and relatively few works have dealt with the forecasting of the solar resources and its application for management of solar-based 
electricity power plants and grid integration strategies. Different approaches to forecast irradiance can be taken depending on the target forecasting time. For very short time forecasts (up to $3 \mathrm{~h}$, now-casting), approaches based on extrapolating the solar radiation field from cloud motion have been proposed [3]. In addition, statistical techniques have been proposed for forecasting solar irradiance with up to $24 \mathrm{~h}$ [4]. However, Numerical Weather Prediction (NWP) models are the basis of solar yield forecasts with up $48 \mathrm{~h}$ time horizon [5], the time range useful for grid integration and decision making in the energy market. NWP models use atmospheric reanalysis initial and boundary conditions for the model run, which are then realistically downscaled (using physical equations) to a better physical resolution (mesoscale model). Because the mesoscale models run over a smaller area than global scale models, the physics can include additional details. Therefore, provided enough computing power, these models can be used to forecast solar irradiance over a wide area with high temporal and spatial resolution.

Earliest evaluation studies on the MM5 mesoscale model [6] reliability for estimating global horizontal irradiance (GHI) were carried out by Zamora et al. in some locations in USA ([7], [8]). Heinemann et al. [3] evaluated the MM5 model GHI forecasts in Germany for lead time up to $48 \mathrm{~h}$. Lorenz et al. [9] evaluated several NWP-based hourly GHI forecasts in Europe. The same author evaluated GHI forecasts, based on the European Centre for Medium-Range Weather Forecast (ECMWF) NWP model, for power prediction of PV systems in Germany [10]. They reported relative root mean square errors (RMSE) values of about $35 \%$ for single stations for a $24 \mathrm{~h}$ horizon forecasts. Remund et al. [11] evaluated different NWP-based GHI forecasts in the USA, reporting relative RMSE values ranging from $20 \%$ to $40 \%$ for a $24 \mathrm{~h}$ forecast horizon. Similar results were reported by Perez et al. [12], evaluating NWP-based irradiance forecasts in several places in the USA. The Weather Research and Forecasting (WRF) model has a wide range of physical parameterizations, which allow setting the model to better describe the physical processes based on model domain, resolution, location and application [13].

\section{WRF presentation}

The weather forecast model is strongly dependent on the characteristic space scale. The meteorology scale encompasses domains ranging from the synoptic, planetary scale, regional or mesoscale domains covering from $5 \mathrm{~km}$ to a few hundred kilometers and below $1 \mathrm{~km}$, the meteorology microscale. The most popular synoptic models are GFS, ECMWF, GME, UKMO while HRM, Hirlam, Lokal Model, WRF-NMM, WRF-ARW, Unified Model, MM5 
are mesoscale models [14]. Usually, the global models provide the initial and boundary conditions needed to start the regional models but they can also be used directly in the local analysis. The WRF model comprises two dynamical nuclei, ARW and NMM, the first stemming from the National Center for Atmospheric Research (NCAR) Advanced Research WRF (ARW) and the other one from the National Center for Environmental Predictions (NCEP). The NMM acronym comes from "non-hydrostatic mesoscale model" while ARW from "advanced research weather forecast system". The WRF model is a multi-grid, multilevel, non-hydrostatic Eulerian model with a wide range of microphysics options.

The popularity of the WRF model has significantly increased even in Europe in the last years. Recently, studies from Spanish meteorological agency have shown that WRF model can be fully integrated as a member of the ensemble forecasting systems [14]. One month simulations of the various physics options of WRF-NMM model using global data from GFS and ECMWF models have shown a relative superiority compared to the results obtained from the Unified Model from UK Meteorological Office and GME from Deutsche Wetterdienst [14].

\section{Radiation model}

The radiation schemes provide atmospheric heating due to radiative flux divergence and surface downward longwave and shortwave radiation for the ground heat budget. All the radiation schemes in WRF currently are column (one-dimensional) schemes, so each column is treated independently, and the fluxes correspond to those in infinite horizontally uniform planes, which is a good approximation if the vertical thickness of the model layers is much less than the horizontal grid length. The short wave radiation microphysics has several options: Eta Geophysical Fluid Dynamics Laboratory (GFDL) version of the Lacis and Hansen parameterization [15]; MM5 based on Dudhia model [16]; Goddard Shortwave based on Chou and Suarez scheme [17]; CAM Shortwave which is spectral-band scheme used in the NCAR Community Atmosphere Model (CAM 3.0).

We have chosen to work with the simplest model MM5 (Dudhia) that has a simple downward integration of solar flux, accounting for clear-air scattering, water vapour absorption and cloud albedo and absorption [15]. It uses look-up tables for clouds from Stephens [18].

The downward component of shortwave flux is evaluated taking into account: 1) the effects of solar zenith angle, which influences the downward component and the path length; 
2) clouds, which have their own albedo and absorption; 3) and clear air, where there is scattering and water-vapour absorption. Thus, the downward shortwave flux

$$
S_{d}(z)=\mu S_{0}-\int_{z}^{t o p}\left(d S_{c s}+d S_{c a}+d S_{a}+d S_{s}\right)
$$

where $\mu$ is the cosine of the zenith angle, $\mathrm{S}_{0}$ is the solar constant, $\mathrm{z}$ is the current level and top the last level of the model. Subscripts "cs" and "ca" refer to cloud absorption and cloud scattering while "a" and "s" to absorption transmisivity and scattering transmisivity. Cloud fraction in a grid box is either 0 or 1 because of the assumed stratiform nature of the clouds. The cloud back-scattering (or albedo) and absorption are bilinearly interpolated from tabulated functions of $\mu$ and $\ln (w / \mu)$ (where $w$ is the vertically integrated cloud water path) derived from Stephens's theoretical results [18]. The total effect of a cloud or multiple layers of cloud above a height $z$ is found from the above function as a percentage of the downward solar flux absorbed or reflected. Then at a height $z-\Delta z$, a new total percentage is calculated from the table allowing the effect of the layer $\Delta z$ to be estimated. However, this percentage is only applied to $\mu S_{0}-\Delta S$ (clear air); that is, the clear-air effect above $z$ is removed. Clear-air water vapour absorption is calculated as a function of water vapour path allowing for solar zenith angle. The absorption function is taken from Lacis and Hansen [15]. The method is a similar integration-difference scheme to that described above for cloud. Clear-air scattering is taken to be uniform and proportional to the atmosphere's mass path length, again allowing for the zenith angle, with a constant giving 20 percent scattering in one atmosphere [6].

\section{Results and discussion}

The radiometric measurement datasets were obtained from the following National Meteorological Administration stations (see Table 1) supplied with Kipp \& Zonen CM6B radiometers.

TABLE 1. Characteristics of ANM Timisoara station

\begin{tabular}{cccccc}
\hline Station name & $\begin{array}{c}\text { Station } \\
\text { code }\end{array}$ & $\begin{array}{c}\text { Geographic } \\
\text { code }\end{array}$ & $\begin{array}{c}\text { Latitude } \\
\text { (deg N) }\end{array}$ & $\begin{array}{c}\text { Longitude } \\
\text { (deg E) }\end{array}$ & $\begin{array}{c}\text { Altitude } \\
\text { (m asl) }\end{array}$ \\
\hline Timisoara & 15247 & 546115 & 45.77 & 21.26 & 86 \\
\hline Cluj & 15120 & 647334 & 46.78 & 23.57 & 417 \\
\hline Jassy & 15090 & 710736 & 47.17 & 27.63 & 103 \\
\hline Galatzi & 15310 & 530801 & 45.47 & 28.03 & 71 \\
\hline Craiova & 15450 & 414352 & 44.31 & 23.87 & 192 \\
\hline
\end{tabular}

The WRF model was operated with the WRF single-moment microphysics scheme following Hong et al. [19], the YSU planetary boundary layer scheme [20], the Kain-Fritsch cumulus scheme [21], MM5 similarity based on Monin-Obukhov with Carslon-Boland 
viscous sub-layer for surface layer ([22], [23], [24]), the unified Noah land-surface model, the RRTM scheme for long-wave radiation [25] and the scheme of Dudhia for shortwave radiation [16]. The synoptic meteorological data started on 06/13/2010-00:00:00 and expanded up to 06/20/2010-12:00:00 covering 180 hours of forecast of the 0 cycle. The simulation domain is centered on the geographical coordinates of the city of Timisoara and expands $1700 \mathrm{~km}$ in E-W direction and $850 \mathrm{~km}$ in N-S direction. The grid spacing is $18.5 \mathrm{~km}$. For this preliminary research we chose not to use nested grids. Two types of comparisons have been performed: one for Timisoara station covering 180 hours of forecasting and the second for the other four stations mentioned in Table 1 over only 72 hours of forecast. The first comparison with measured data is illustrated in Fig. 1. Due to partial lack of measured data there are less than 180 recordings that have been taken into account.
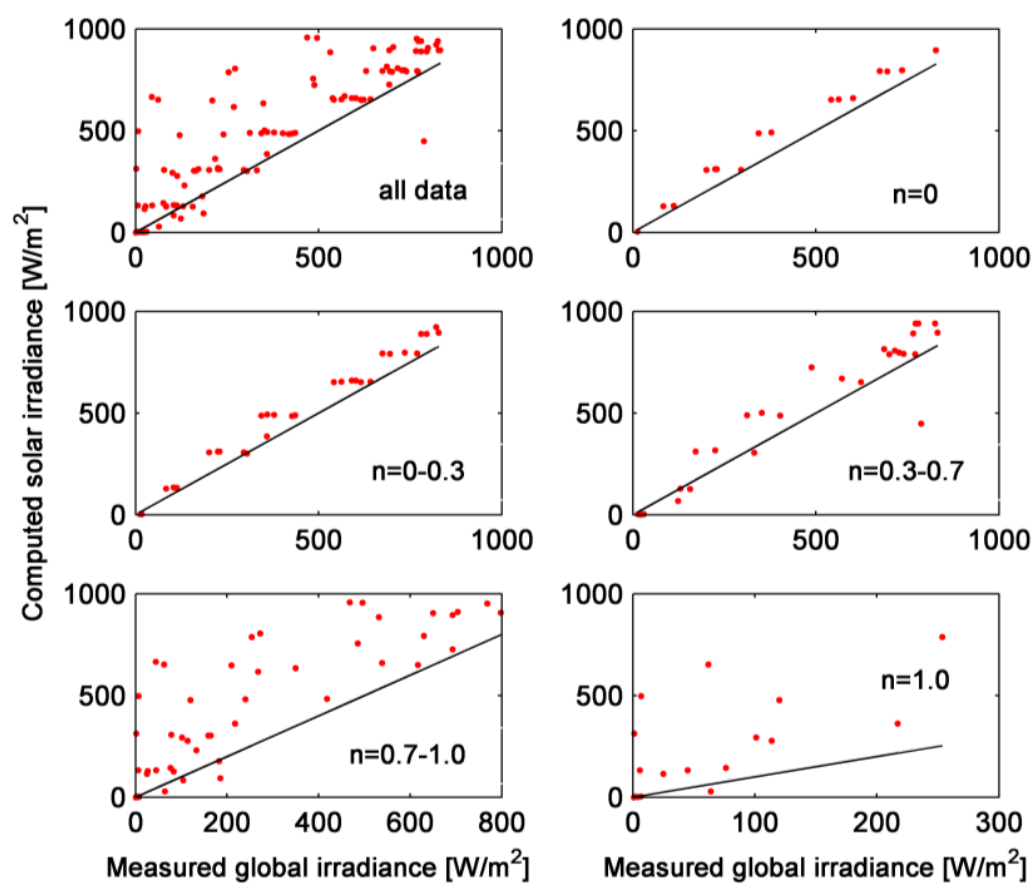

Fig. 1. Computed vs. measured global irradiance for Timisoara station. (n denotes the cloudiness class)

The results of the statistical analysis are comparable with those reported in literature [26], although some input data were different (e.g. spatial resolution). The influence of cloudiness on the global solar irradiance statistics for Timisoara station is presented in Table 2.

TABLE 2. Relative statistics distribution over cloudiness classes for Timisoara station.

\begin{tabular}{ccccccc}
\hline & $\mathrm{n}=0$ & $\mathrm{n}=0-0.3$ & $\mathrm{n}=0.3-0.7$ & $\mathrm{n}=0.7-0.1$ & $\mathrm{n}=1.0$ & all data \\
\hline rMBE $(\%)$ & 18.23 & 14.22 & 11.68 & 75.69 & 281.91 & 32.50 \\
\hline rRMSE (\%) & 21.62 & 17.64 & 26.55 & 105 & 412.84 & 53.63 \\
\hline
\end{tabular}


The outcome of the second comparison is depicted in Figs. 2-3 and the associated statistics in Table 3. The obvious result is that the shorter the forecast is the better correlation with measurements is obtained. Taking into account the large number of possible combinations of various physical options in WRF together with the spatial grid and time resolution, it becomes necessary a supplementary, more detailed analysis in order to decide which is the optimal set of switches in WRF input file that enables us to extend the solar radiation forecast even for high degree of cloudiness. On the other hand, as results in Table 3 perfectly illustrate, it seems that the local surface physics model might have an important role on the numerical simulation of global solar irradiance.
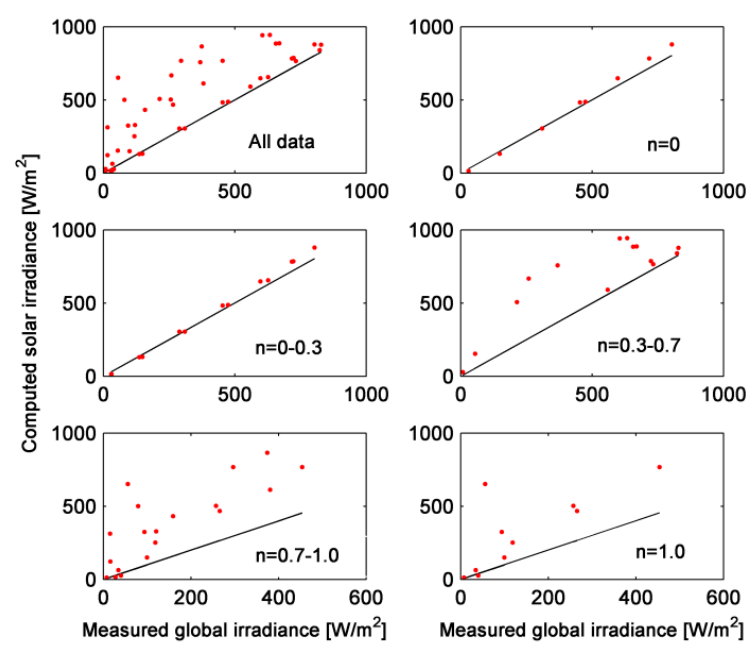

a)
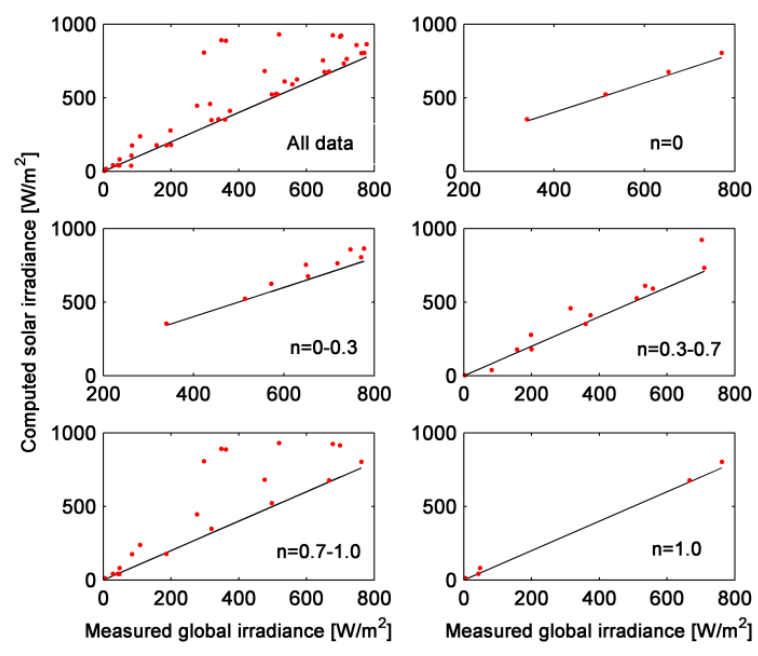

b)

Fig. 2. Computed vs. measured global irradiance for a) Cluj; b) Jassy meteorological stations.

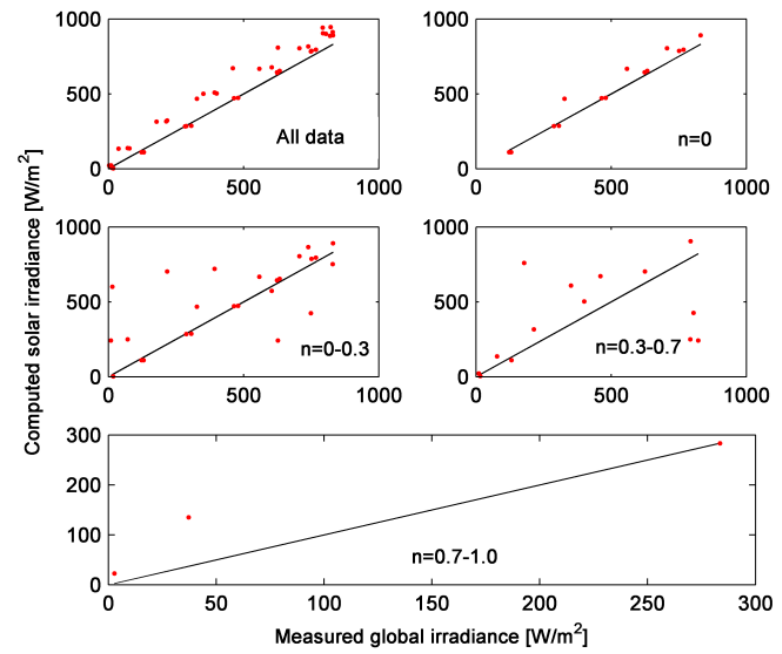

a)

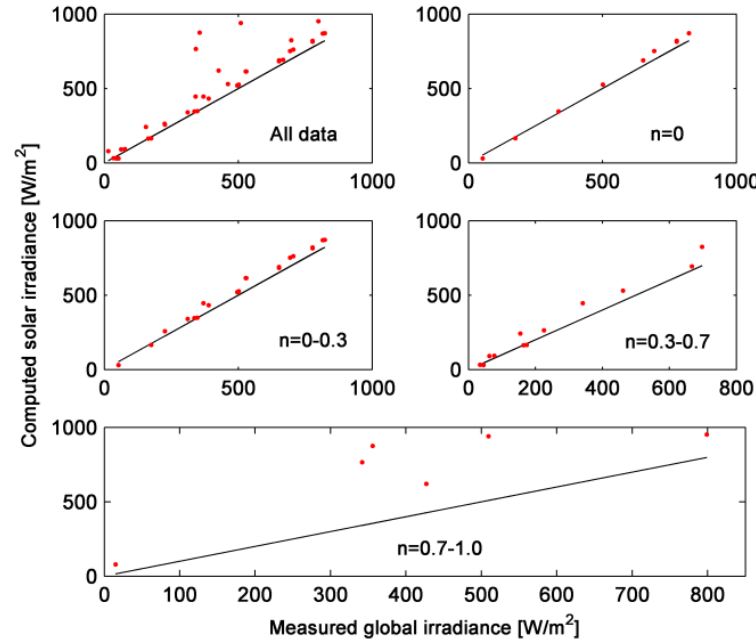

b)

Fig. 3. Computed vs. measured global irradiance for a) Craiova; b) Galatzi meteorological stations. 
TABEL 3. Relative statistics distribution over cloudiness classes for $72 \mathrm{~h}$ forecast

\begin{tabular}{cccccccc}
\hline \multirow{2}{*}{ tation } & & \multicolumn{7}{c}{ Cloudiness Classes } \\
\cline { 3 - 8 } & & $\mathrm{n}=0$ & $\mathrm{n}=0-0.3$ & $\mathrm{n}=0.3-0.7$ & $\mathrm{n}=0.7-0.1$ & $\mathrm{n}=1.0$ & all data \\
\hline \multirow{2}{*}{ Cluj } & rMBE (\%) & 5.60 & 5.24 & 35.04 & 148.05 & 125.95 & 45.96 \\
\cline { 2 - 8 } & $\mathrm{rRMSE}(\%)$ & 9.59 & 9.41 & 44.94 & 186.99 & 176.04 & 66.85 \\
\hline \multirow{2}{*}{ Craiova } & $\mathrm{rMBE}(\%)$ & 6.57 & 13.90 & 0.28 & 36.34 & $\mathrm{NA}$ & 14.44 \\
\cline { 2 - 8 } & $\mathrm{rRMSE}(\%)$ & 11.87 & 46.51 & 74.19 & 53.45 & $\mathrm{NA}$ & 20.42 \\
\hline \multirow{2}{*}{ Iasi } & rMBE(\%) & 3.45 & 8.31 & 12.17 & 49.41 & 5.98 & 25.13 \\
\cline { 2 - 8 } & rRMSE(\%) & 3.84 & 10.09 & 22.56 & 76.17 & 7.95 & 45.07 \\
\hline \multirow{2}{*}{ Galati } & rMBE(\%) & 4.80 & 7.36 & 15.34 & 73.05 & $\mathrm{NA}$ & 19.17 \\
\cline { 2 - 8 } & rRMSE(\%) & 6.81 & 9.19 & 23.29 & 83.88 & $\mathrm{NA}$ & 34.87 \\
\hline
\end{tabular}

\section{Conclusions}

Our preliminary analysis shows that the computed global solar irradiance fits quite well within the experimental data for situations ranging from clear sky $(n=0)$ to moderate pointcloudiness index $(n \in[0,0.7])$ (see Fig. 1-3). The statistics presented in Tabels 2-3 show five to ten percent larger values for the mean bias error (rMBE) compared with the results of LaraFanego [26]. This is most probably due to different microphysics options and to the coarser grid used. The chosen WRF microphysics model and its interactions cannot reasonably predict situations characterized by strong cloud covering. Further tests have to be performed in order to fully evaluate the possibility of solar radiation forecast.

\section{Acknowledgments}

This work was supported by a grant of the Romanian National Authority for Scientific Research, CNCS - UEFISCDI, project number PN-II-ID-PCE-2011-3-0089 and by the European Cooperation in Science and Technology project COST ES1002.

\section{References}

[1] V. Badescu and A. Dumitrescu, "Testing Magicsol under the climate and latitudes of Romania", COST Action ES1002 "WIRE", Workshop on Remote Sensing Measurements for Renewable Energy, Technical University of Denmark DTU, Wind Energy Department Riso campus, Roskilde, Denmark, May 22-23, 2012.

[2] L. Martin, L. F. Zarzalejo, J. Polo, A. Navarro, R. Marchante, M. Cony, "Prediction of global solar irradiance based on time series analysis: Application to solar thermal power plants energy production planning", Solar Energy, 84 (2010) 1772-1781,

[3] D. Heinemann, E. Lorenz and M. Girodo, Forecasting of solar radiation in: Dunlop, E.D., Wald, L., Suri, M. (Eds.), Solar Energy Resource Management for Electricity Generation from Local Level to Global Scale. Nova Science Publishers, Hauppauge, 2006. 
[4] A. Mellit and A.M. Pavan, Sol. Energy 84 (5) (2010) 807-821.

[5] IEA, Energy Technologies at the Cutting Edge, International Energy Agency, OECD Publication Service, OECD, Paris, 2007.

[6] G. Grell, J. Dudhia, and D. Stauffer, "A Description of the Fifth-Generation Penn State/NCAR Mesoscale Model (MM5)", NCAR Tech. Note, NCAR/TN-398+STR, USA, 1998.

[7] R.J. Zamora, E.G. Dutton, M. Trainer, S.A. McKeen, J.M. Wilczak, Y.T. Hou, Mon. Weather Rev. 133 (2005) 783-792.

[8] R.J. Zamora, S. Solomon, E.G. Dutton, J.W. Bao, M. Trainer, R.W. Portmann, A.B. White, D.W. Nelson, R.T. McNider, J. Geophys.Res. 108 (D2) (2003) 4050.

[9] E. Lorenz, J. Remund, S.C. Muller, W. Traunmuller, G. Steinmaurer, D. Pozo, J.A. RuizArias, V.L. Fanego, L. Ramirez, M.G. Romeo, C. Kurz, L.M. Pomares, C.G. Guerrero, Benchmarking of different approaches to forecast solar irradiance. In: 24th European Photovoltaic Solar Energy Conference, Hamburg, Germany, 21-25 September 2009.

[10]E. Lorenz, J. Hurka, D. Heinemann, H.G. Beyer, IEEE J. Selected Topics Appl. Earth Observations Remote Sens. 2 (1) (2009).

[11]R. Remund, Y. Perez, E. Lorenz, Comparison of solar radiation forecasts for the USA. In: Proceedings of the 23rd European Photovoltaic Solar Energy Conference, Valencia, Spain, (2008) 1.9-4.9.

[12]Y. Perez and F.J. Ramos-Real, Renew. Sust. Energy Rev., 13, 1058-1066, 2009.

[13] J.A. Ruiz-Arias, D. Pozo-Vazquez, N. Sanchez-Sanchez, J.P. Montavez, A. Hayas-Barru, J. Tovar-Pescador, Il Nuovo Cimento, 31 (5-6) (2008) 825-842.

[14]D. Santos-Muñoz, J. Wolff, C. Santos, J.A. García-Moya and L. Nance, Implementation and validation of WRF model as ensemble member of a probabilistic prediction system over Europe. In: 10th Annual WRF Users' Workshop, 23-26 June 2009 in Boulder, CO, (2009).

[15]A. A. Lacis and J. E. Hansen, "A parameterization for the absorption of solar radiation in the earth's atmosphere", J. Atmos. Sci., 31 (1974) 118-133.

[16]J. Dudhia, "Numerical study of convection observed during the winter monsoon experiment using a mesoscale two-dimensional model", J. Atmos. Sci., 46 (1989) 30773107.

[17] M.-D. Chou and M. J. Suarez, An efficient thermal infrared radiation parameterization for use in general circulation models. NASA Tech. Memo. 104606, 3 (1994) 85.

[18] G. L. Stephens, "Radiation profiles in extended water clouds. Part II: Parameterization schemes", J. Atmos. Sci., 35 (1978) 2123-2132. 
[19] S.-Y. Hong, J. Dudhia and S.-H. Chen, "A Revised Approach to Ice Microphysical Processes for the Bulk Parameterization of Clouds and Precipitation", Mon. Wea. Rev., 132 (2004) 103-120.

[20] S.-Y. Hong, Y. Noh and J. Dudhia, "A new vertical diffusion package with an explicit treatment of entrainment processes", Mon. Wea. Rev., 134 (2006) 2318-2341.

[21]J. S. Kain and J. M. Fritsch, Convective parameterization for mesoscale models: The Kain-Fritcsh scheme, The representation of cumulus convection in numerical models. In: K. A.Emanuel and D.J. Raymond, Eds., Amer. Meteor. Soc., (1993) 246.

[22]C. A. Paulson, "The mathematical representation of wind speed and temperature profiles in the unstable atmospheric surface layer", J. Appl. Meteor., 9 (1970) 857-861.

[23]A. J. Dyer and B. B. Hicks, "Flux-gradient relationships in the constant flux layer", Quart. J. Roy. Meteor. Soc., 96 (1970) 715-721.

[24]E. K. Webb, "Profile relationships: The log-linear range, and extension to strong stability", Quart. J. Roy. Meteor. Soc., 96 (1970) 67-90.

[25]E. J. Mlawer, S. J. Taubman, P. D. Brown, M. J. Iacono and S. A. Clough, "Radiative transfer for inhomogeneous atmosphere: RRTM, a validated correlated-k model for the longwave", J. Geophys. Res., 102 (D14) (1997) 16663-16682.

[26] V. Lara-Fanego, J.A. Ruiz-Arias, D. Pozo-Vazquez, F.J. Santos-Alamillos, J. TovarPescador, "Evaluation of the WRF model solar irradiance forecasts in Andalusia (southern Spain)", Solar Energy (2011) doi:10.1016/j.solener.2011.02.014. 